\title{
Delocalization of states in two-component superlattices with correlated disorder
}

\author{
T. Hakobyan \\ Yerevan Physics Institute, Alikhanian Brothers Street 2, 375036 Yerevan, Armenia \\ D. Sedrakyan \\ Yerevan State University, Alex Manoogian Street, Yerevan, Armenia \\ A. Sedrakyan* \\ Yerevan Physics Institute, Alikhanian Brothers Street 2, 375036 Yerevan, Armenia \\ I. Gómez and F. Domínguez-Adame \\ GSIC, Departamento de Física de Materiales, Universidad Complutense, E-28040 Madrid, Spain
}

(Received 29 November 1999)

\begin{abstract}
Electron and phonon states in two different models of intentionally disordered superlattices are studied analytically as well as numerically. The localization length is calculated exactly and we found that it diverges for particular energies or frequencies, suggesting the existence of delocalized states for both electrons and phonons. Numerical calculations for the transmission coefficient support the existence of these delocalized states.
\end{abstract}

\section{INTRODUCTION}

Since a remarkable article by Anderson, ${ }^{1}$ the problem of localization of particles in systems with random distribution of parameters is still of continuous interest for physicists. It was conjectured by Mott and Twose, ${ }^{2}$ rigorously proved for some systems ${ }^{4}$ and then generally argued in Ref. 3 that, in a case of full randomness of the parameters of the model, all states are localized in one and two dimensions. However, there exist several exceptions to this rule. These exceptions are mainly related to the existence of correlations, either in disorder or between the quasiparticles of the system, as well as anomalous (nonexponential) localization found at specific regions of the energy spectrum. Recently the interest in the investigations of the conditions for breaking of Anderson localization due to correlations in the disorder has increased substantially. Evidences were found, that in a presence of internal correlations in disordered systems delocalized (extended) states may appear. ${ }^{5-21}$ Due to the lack of experimental confirmations, there are some controversies around the importance of these results and their physical applications. That is one of the reasons why the experimental evidence of extended states, found in the studies of the electronic properties of GaAs-AlGaAs superlattice (SL) with intentional correlated disorder by means of photoluminescence and vertical $d_{c}$ resistance, ${ }^{22}$ looks promising.

Following this line of work, here we consider twocomponent SL's with particular types of correlated disorder for thickness of the layers. We demonstrate the appearance of delocalized states for phonon as well as for electron transport problems. Following the technique developed in Ref. 23 we find exactly the transfer matrix for scatterers on the boundaries of the layers, and calculate the localization length and dimensionless Landauer resistance, which allows us to determine the energy (or frequency) of the resonant states for which delocalization occurs. Two types of disorder for the thickness of the layers will be considered in the paper, namely (i) the thickness of one of the SL components (referred to as $A$ layers) is fixed and equal to $d_{1}$, while the thickness of the other component ( $B$ layers) is randomly distributed with probability

$$
g(y)= \begin{cases}\frac{1}{d_{i}}, & 0<y<d_{i}, \\ 0, & \text { otherwise }\end{cases}
$$

(ii) Again, the thickness of the $A$ layers is set to $d_{1}$, while for $B$ layers we take a sequence of fixed and randomly distributed thicknesses. In other words, we take following distribution of layers $A$ (fixed) $B$ (fixed) $A$ (fixed) $B$ (random) . . . .

In both cases, conditions on energies (and frequencies) of delocalized states are found and it is easy to see that they can be fulfilled. We think that these two types of disorder are easy to organize in samples grown by molecular-beam epitaxy (MBE) and experimentally check the existence of extended states for both electrons and phonons, within the spirit of Ref. 22.

\section{TRANSFER MATRIX AND LANDAUER RESISTANCE}

Let us consider a SL consisting of two component materials $(A$ and $B)$, grown in the $x$ direction with the thicknesses of the layers $\Delta x_{i}=x_{i}-x_{i-1}$, with $i=1,2, \ldots, 2 N$ and $x_{i}$ 's being the coordinate of the boundaries between the layers. We will investigate the propagation of particles and their localization along the grow direction $x$. The wave equation for transversal phonon displacement $u(x, y, t)$ is

$$
\frac{\partial^{2} u(x, y, t)}{\partial t^{2}}-c_{t}^{2} \Delta u(x, y, t)=0,
$$


where the velocity of sound $c_{t}$ is defined by the density of matter $\rho$ and modulus of rigidity $\mu$ as $c_{t}^{2}=\mu / \rho$.

Solutions of the wave Eq. (2) with frequency $\omega$ are a superposition of forward- and backward-scattering waves and can be represented as follows:

$$
\begin{gathered}
u_{2 n-1}(x, y, t)=\left(c_{2 n-1} e^{i k_{1}\left(x-x_{2 n-2}\right)}\right. \\
\left.+\bar{c}_{2 n-1} e^{-i k_{1}\left(x-x_{2 n-2}\right)}\right) e^{i w t} \\
u_{2 n}(x, y, t)=\left(c_{2 n} e^{i k_{2}\left(x-x_{2 n-1}\right)}+\bar{c}_{2 n} e^{-i k_{1}\left(x-x_{2 n-1}\right)}\right) e^{i w t}, \\
n=1, \ldots, N,
\end{gathered}
$$

where we have defined

where for the wave vector $k_{i}, i=1,2$ we have

$$
k_{i}^{2}=\frac{\omega^{2}}{c_{i t}^{2}} .
$$

Here $c_{i t}$ is the velocity of sound in media $i$; quantities with $i=1$ correspond to those of material $A$ and, in the same way, quantities with $i=2$ correspond to those of material $B$. In Eqs. (3) $2 n$ (respectively, $2 n-1$ ) numerates the layers $B$ (respectively, $A$ ).

We should now impose the boundary conditions on the solutions (3) for displacements $u_{2 n}$ and $u_{2 n-1}$, as it was demonstrated in Ref. 23,

$$
\begin{gathered}
\mu_{2} \partial_{x} u_{2 n}\left(x_{2 n-1}\right)=\mu_{1} \partial_{x} u_{2 n-1}\left(x_{2 n-1}\right), \\
u_{2 n}\left(x_{2 n-1}\right)=u_{2 n-1}\left(x_{2 n-1}\right) n=1,2, \ldots N,
\end{gathered}
$$

which are nothing but continuity conditions on the displacements $u_{i}(x, y, t)$ and the forces $\mu_{i} \partial_{x} u_{i}(x, y, t)$ at the boundaries of the layers.

$$
\psi_{i}=\left(\begin{array}{c}
c_{i} \\
\bar{c}_{i}
\end{array}\right), \quad i=1,2, \ldots, 2 N
$$

The solution of the boundary conditions (5) allows us to express linearly all amplitudes $c_{i}$ and $\bar{c}_{i}$ of scattering modes in the slice $i$ through amplitudes of the initial wave $c_{1}$ and $\bar{c}_{1}$ as follows:

$$
\psi_{2 n}=\prod_{j=1}^{n} T_{j} \psi_{1}=T \psi_{1},
$$

The expression of the transfer matrix $T_{j}$

$$
T_{j}=T_{2 j} T_{2 j-1}=\left(\begin{array}{cc}
\alpha_{j} & \beta_{j} \\
\beta_{j}^{*} & \alpha_{j}^{*}
\end{array}\right)
$$

with

$$
\begin{aligned}
& \alpha=\left[\frac{i}{2}\left(\frac{\mu_{1} k_{1}}{\mu_{2} k_{2}}+\frac{\mu_{2} k_{2}}{\mu_{1} k_{1}}\right) \sin k_{1}\left(x_{2 j-1}-x_{2 j-2}\right)\right. \\
&\left.+\cos k_{1}\left(x_{2 j-1}-x_{2 j-2}\right)\right] e^{i k_{2}\left(x_{2 j-2}-x_{2 j-3}\right)}, \\
& \beta=\frac{i}{2}\left(\frac{\mu_{1} k_{1}}{\mu_{2} k_{2}}-\frac{\mu_{2} k_{2}}{\mu_{1} k_{1}}\right) \sin k_{1}\left(x_{2 j-1}-x_{2 j-2}\right) \\
& \times e^{-i k_{2}\left(x_{2 j-2}-x_{2 j-3}\right)},
\end{aligned}
$$

is easy to obtain performing the product of $T_{2 j}$ and $T_{2 j-1}$ matrices

$$
\begin{aligned}
& T_{2 j}=\frac{1}{2 \mu_{2} k_{2}}\left(\begin{array}{ll}
\left(\mu_{1} k_{1}+\mu_{2} k_{2}\right) e^{i k_{1}\left(x_{2 j-1}-x_{2 j-2}\right)} & \left(\mu_{2} k_{2}-\mu_{1} k_{1}\right) e^{-i k_{1}\left(x_{2 j-1}-x_{2 j-2}\right)} \\
\left(\mu_{2} k_{2}-\mu_{1} k_{1}\right) e^{i k_{1}\left(x_{2 j-1}-x_{2 j-2}\right)} & \left(\mu_{1} k_{1}+\mu_{2} k_{2}\right) e^{-i k_{1}\left(x_{2 j-1}-x_{2 j-2}\right)}
\end{array}\right), \\
& T_{2 j-1}=\frac{1}{2 \mu_{1} k_{1}}\left(\begin{array}{ll}
\left(\mu_{1} k_{1}+\mu_{2} k_{2}\right) e^{i k_{2}\left(x_{2 j-2}-x_{2 j-3}\right)} & \left(\mu_{1} k_{1}-\mu_{2} k_{2}\right) e^{-i k_{2}\left(x_{2 j-2}-x_{2 j-3}\right)} \\
\left(\mu_{1} k_{1}-\mu_{2} k_{2}\right) e^{i k_{2}\left(x_{2 j-2}-x_{2 j-3}\right)} & \left(\mu_{1} k_{1}+\mu_{2} k_{2}\right) e^{-i k_{2}\left(x_{2 j-2}-x_{2 j-3}\right)}
\end{array}\right) \text {. }
\end{aligned}
$$

If we now focus on a model where electrons with effective mass $m_{i}$ and potential energy $V_{i}$ at layer $A(i=1)$ or $B(I=2)$ impinge on the SL, then it is necessary to change the equation of motion (3) for phonons by the Schrödinger equation for the envelope function $\left(\hbar=1\right.$ and $\vec{k}_{\perp}=0$ hereafter):

$$
i \frac{\partial u(x, t)}{\partial t}+\left(\frac{1}{2 m_{i}} \Delta-V_{i}\right) u(x, t)=0, \quad i=1,2, \ldots, 2 N,
$$

but the form of general solution (3) is valid provided

$$
k_{i}^{2}=2 m_{i}\left(E-V_{i}\right)
$$

Analogously, the boundary conditions (5) now will read as

$$
u_{2 n}\left(x_{2 n-1}\right)=u_{2 n-1}\left(x_{2 n-1}\right) \text {, }
$$$$
\frac{1}{m_{2}} \partial_{x} u_{2 n}\left(x_{2 n-1}\right)=\frac{1}{m_{1}} \partial_{x} u_{2 n-1}\left(x_{2 n-1}\right), \quad n=1,2, \ldots, N
$$

It is a matter of simple algebra to see from Eqs. (12) and (13) that the transfer matrix (8) for the electron problem will have the same form as for phonons in the expression (8) but replacing $\mu_{i} \rightarrow 1 / m_{i}$.

In Ref. 26 the problem of transport of particles in the one-dimensional space for a wide class of disordered models was considered within the transfer-matrix approach and gen- 
eral results were obtained. It was demonstrated that the transfer matrix of one-dimensional problems belongs to $\operatorname{SL}(2, R)$ group and randomness of media can be exactly taken into account for such quantities as the Landauer resistance. ${ }^{24}$ It is easy to see that our transfer matrix also belongs to $\operatorname{SL}(2, R)$ group.

Following Refs. 24-26 let us now define the dimensionless Landauer resistance as the ratio of reflection and transmission coefficients

$$
\rho=\left|\frac{r}{\tau}\right|^{*}=\frac{1-|\tau|^{2}}{|\tau|^{2}}=T_{12} T_{21}^{*} .
$$

In order to proceed further we should use the relation for the direct product $T \otimes T^{-1}$ presented in Ref. 23:

$$
\left(T_{j}\right)_{\alpha^{\prime}}^{\alpha}\left(T_{j}^{-1}\right)_{\beta}^{\beta^{\prime}}=\frac{1}{2}(\delta)_{\beta}^{\alpha}(\delta)_{\alpha^{\prime}}^{\beta^{\prime}}+\frac{1}{2}\left(\sigma^{\mu}\right)_{\alpha^{\prime}}^{\beta^{\prime}} \Lambda_{j}^{\mu \nu}\left(\sigma^{\nu}\right)_{\beta}^{\alpha},
$$

where

$$
\Lambda_{j}^{\mu \nu}=\frac{1}{2} \operatorname{Tr}\left(T_{j} \sigma^{\mu} T_{j}^{-1} \sigma^{\nu}\right)
$$

is the spin-one part of the direct product. But for Landauer resistance we need to calculate $T \otimes T^{+}$. It is easy to see from Eq. (8) that

$$
\sigma_{3} T^{-1} \sigma_{3}=T^{\dagger} \text {. }
$$

Therefore by multiplying Eq. (15) from the left and right by $\sigma_{3}$ we will have

$$
\left(T_{j}\right)_{\alpha^{\prime}}^{\alpha}\left(T_{j}^{+}\right)_{\beta}^{\beta^{\prime}}=\frac{1}{2}\left(\sigma_{3}\right)_{\beta}^{\alpha}\left(\sigma_{3}\right)_{\alpha^{\prime}}^{\beta^{\prime}}+\frac{1}{2}\left(\sigma^{\mu} \sigma_{3}\right)_{\alpha^{\prime}}^{\beta^{\prime}} \Lambda_{j}^{\mu \nu}\left(\sigma^{\nu} \sigma_{3}\right)_{\beta}^{\alpha} .
$$

It is straightforward now to calculate the Landauer resistance $\rho$ by using formulas (14)-(18), which seem to depend only on $(3,3)$ element of the product of the transfer matrices,

$$
\rho=\frac{1}{2}\left[-1+\left(\prod_{j=1}^{N} \Lambda_{j}\right)^{33}\right] .
$$

This expression is of remarkable interest because it is multiplicative in $\Lambda_{j}^{\mu \nu}(j=1,2, \ldots, N)$, each of which depends only on local parameters (thickness and the other model parameters) of the $j$ th pair of layers. Therefore this expression for the Landauer resistance becomes valid for media with arbitrary distribution of the parameters.

\section{CONDITIONS FOR THE EXISTENCE OF EXTENDED STATES}

As we see from the expression (19), due to the multiplicative form of the dependence of the Landauer resistance on local parameters, its average over different type of correlated disorder can be easily taken. We should simply average $\Lambda_{i}^{\mu \nu}$ in each layer separately and then take the product of them. Now we will consider the two types of correlated disorder mentioned in the Introduction.

Let us take fixed thickness for the $A$ layers of the SL as $d_{1}$ and a random distribution of thicknesses for the component
$B$ by use of the probability distribution (1). Simple substitution of expression (8) for the transfer matrix $T$ into the formula (16) for $\Lambda_{j}^{\mu \nu}$ gives

$$
\begin{aligned}
\Lambda_{j}^{11}= & \cos \left[2 k_{1} d_{1}^{j}\right] \cos \left[2 k_{2} d_{2}^{j}\right] \\
& -\cosh [\theta] \sin \left[2 k_{2} d_{2}^{j}\right] \sin \left[2 k_{1} d_{1}^{j}\right],
\end{aligned}
$$

$$
\begin{aligned}
\Lambda_{j}^{12}= & \left(\cosh ^{2}[\theta] \cos \left[2 k_{1} d_{1}^{j}\right]-\sinh ^{2}[\theta]\right) \sin \left[2 k_{2} d_{2}^{j}\right] \\
+ & \cosh [\theta] \cos \left[2 k_{2} d_{2}^{j}\right] \sin \left[2 k_{1} d_{1}^{j}\right], \\
\Lambda_{j}^{13}= & -i \sinh [\theta]\left\{\cos \left[2 k_{2} d_{2}^{j}\right] \sin \left[2 k_{1} d_{1}^{j}\right]\right. \\
& \left.-\cosh [\theta]\left(1-\cos \left[2 k_{1} d_{1}^{j}\right]\right) \sin \left[2 k_{2} d_{2}^{j}\right]\right\}, \\
\Lambda_{j}^{21}= & -\sin \left[2 k_{2} d_{2}^{j}\right] \cos \left[2 k_{1} d_{1}^{j}\right] \\
& -\cosh [\theta] \cos \left[2 k_{2} d_{2}^{j}\right] \sin \left[2 k_{1} d_{1}^{j}\right],
\end{aligned}
$$$$
\Lambda_{j}^{22}=\left(\cosh ^{2}[\theta] \cos \left[2 k_{1} d_{1}^{j}\right]-\sinh ^{2}[\theta]\right) \cos \left[2 k_{2} d_{2}^{j}\right]
$$$$
-\cosh [\theta] \sin \left[2 k_{1} d_{1}^{j}\right] \sin \left[2 k_{2} d_{2}^{j}\right] \text {, }
$$$$
\Lambda_{j}^{23}=i \sinh [\theta]\left\{\sin \left[2 k_{1} d_{1}^{j}\right] \sin \left[2 k_{2} d_{2}^{j}\right]\right.
$$$$
\left.+\cosh [\theta]\left(1-\cos \left[2 k_{1} d_{1}^{j}\right]\right)\right\}
$$$$
\Lambda_{j}^{31}=i \sinh [\theta] \sin \left[2 k_{1} d_{1}^{j}\right],
$$$$
\Lambda_{j}^{32}=i \sinh [2 \theta]\left(1-\cos \left[2 k_{1} d_{1}^{j}\right]\right) / 2,
$$$$
\Lambda_{j}^{33}=\cosh ^{2}[\theta]-\sinh ^{2}[\theta] \cos \left[2 k_{1} d_{1}^{j}\right],
$$

where $\theta$ is defined by

$$
\cosh [\theta]=\frac{1}{2}\left(\frac{\mu_{1} k_{1}}{\mu_{2} k_{2}}+\frac{\mu_{2} k_{2}}{\mu_{1} k_{1}}\right)
$$

and $d_{1}^{j}=x_{2 j-1}-x_{2 j-2}$ and $d_{2}^{j}=x_{2 j-2}-x_{2 j-3}$ are the thicknesses of the $j$ th pair of layers.

Now we should fix $d_{1}^{j}=d_{1}$ for the component $A$ and take the average over $d_{2}^{j}$ using the probability distribution (1), which will give $\langle\Lambda\rangle^{\mu \nu}$ defined by the expressions (20), but where $\cos \left[2 k_{2} d_{2}^{j}\right]$ and $\sin \left[2 k_{2} d_{2}^{j}\right]$ are changed by their average values

$$
\begin{gathered}
a=\left\langle\cos \left[2 k_{2} d_{2}^{j}\right]\right\rangle=\frac{\sin \left[2 d_{2} k_{2}\right]}{2 d_{2} k_{2}}, \\
b=\left\langle\sin \left[2 k_{2} d_{2}^{j}\right]\right\rangle=\frac{\sin ^{2}\left[d_{2} k_{2}\right]}{d_{2} k_{2}} .
\end{gathered}
$$

Then, for the averaged Landauer resistance we will have

$$
\langle\rho\rangle=\frac{1}{2}\left[-1+\left(\langle\Lambda\rangle^{N}\right)^{33}\right] .
$$

For large sample sizes $(N \gg 1)$, as it was first argued in Refs. 24 and 27 that the resistance should behave as $e^{\gamma N}$, where the Lyapunov exponent $\gamma$ provides the phonon correlation length. Using Eq. (23) and the definition of Lyapunov exponent $\gamma=\lim _{N \rightarrow \infty} \ln \rho / N$ we can find an exact expression for localization length 


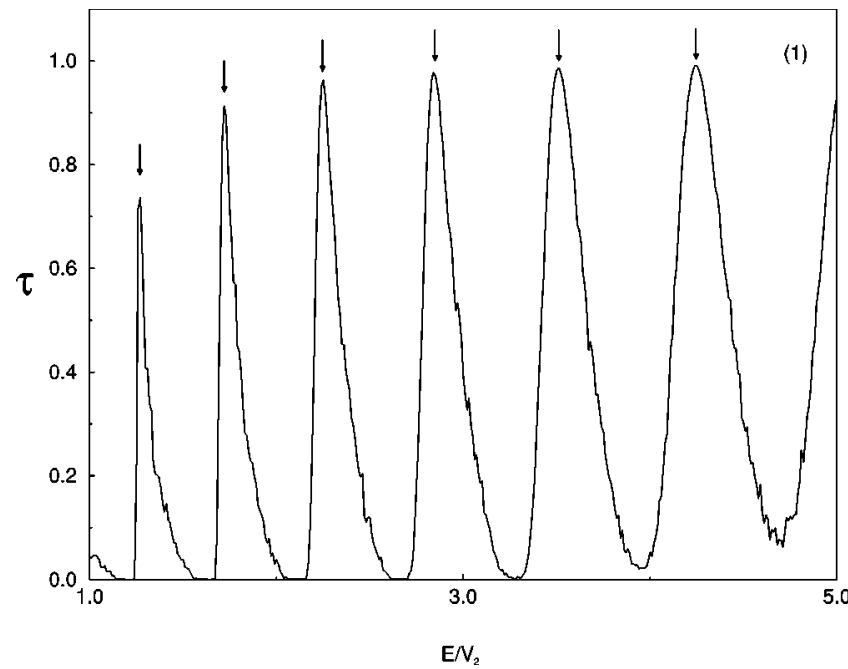

FIG. 1. Transmission coefficient $\tau$ as a function of the reduced energy $E / V_{2}$. The arrows show the energies given in Eq. (29). The height and the nominal width of the barriers are, respectively, $V_{2}$ $=0.4 \mathrm{eV}$ and $d_{2}=15 \AA$, the width of the wells is $d_{1}=200 \AA$, and the number of periods is $N=200$.

$$
\xi^{-1}=\ln \lambda,
$$

where $\lambda$ is the closest to one eigenvalue of the matrix $\left\langle\Lambda^{33}\right\rangle$. Excitations are localized or not depending on the behavior of $\xi$. If at some frequency $\omega_{c}$ (or energy) the localization length becomes infinite, we generally have delocalized states ${ }^{28}$ and the expression (24) shows that it will occur when $\lambda\left(\omega_{c}\right)$ $=1$. Therefore we should elucidate whether the matrix $\langle\Lambda\rangle$ can support unity eigenvalue or not. It is then necessary to calculate the determinant of the matrix $1-\langle\Lambda\rangle$,

$$
\operatorname{det}[1-\langle\Lambda\rangle]=\frac{1}{2} \sin ^{2}\left(d_{1} k_{1}\right)\left(\frac{\mu_{1} k_{1}}{\mu_{2} k_{2}}-\frac{\mu_{2} k_{2}}{\mu_{1} k_{1}}\right)^{2}\left(a^{2}+b^{2}-1\right),
$$

from where it follows that the condition to have an extended state is

$$
\sin \left(d_{1} k_{1}\right)=0
$$

Let us now fix the thickness of the component $A$ of the SL as $d_{1}$ and for the component $B$ take fixed and random thicknesses in a sequence. Then the extended states can appear when

$$
\operatorname{det}[1-\Lambda\langle\Lambda\rangle]=0,
$$

where $\Lambda$ is matrix (20) with fixed thickness of $B$ layers $d_{2}^{j}$ $=d_{2}$. It turns out that the condition (27) is equivalent to the equation

$$
\cos k_{1} d_{1} \cos k_{2} d_{2}-\frac{1}{2}\left(\frac{\mu_{1} k_{1}}{\mu_{2} k_{2}}+\frac{\mu_{2} k_{2}}{\mu_{1} k_{1}}\right) \sin k_{1} d_{1} \sin k_{2} d_{2}=0 .
$$

\section{NUMERICAL RESULTS}

In order to validate the results of our previous formalism, we performed some numerical calculations which allow us to show the existence of the extended states discussed above.

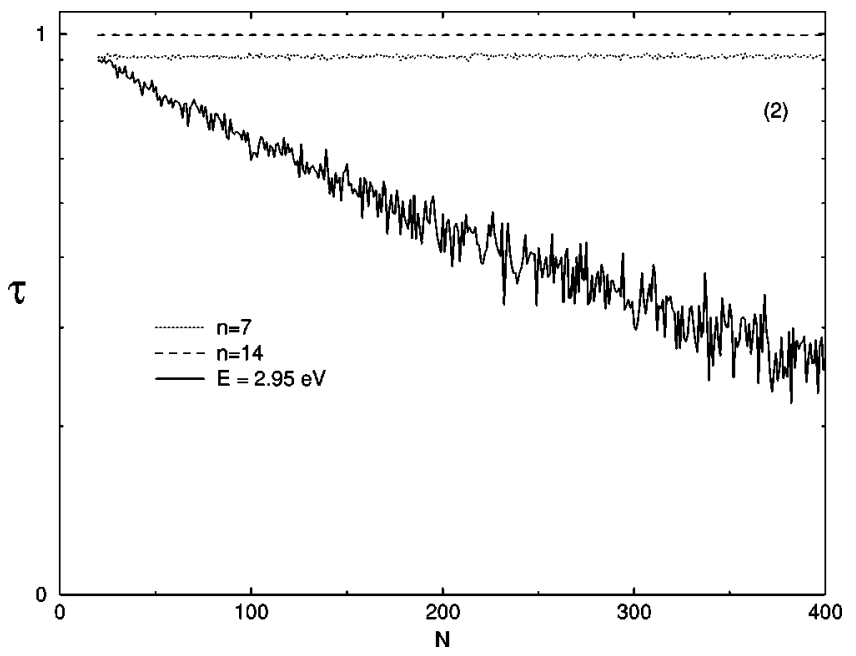

FIG. 2. Transmission coefficient $\tau$ as a function of the system size $N$. The dotted and dashed lines correspond, respectively, to $n$ $=7$ and $n=14$ in Eq. (29). The solid line corresponds to an energy between $n=14$ and $n=15$. Structural parameters are the same as in the previous figure.

We will focus our attention on the electronic model of disorder we previously referred to as model (i), and calculate for that kind of disorder the transmission coefficient as a function of energy, as well as a function of the system size when the energy is fixed to one of that given by expression (26). The transmission coefficient was numerically computed using the transfer-matrix formalism. ${ }^{29,30}$

Figure 1 shows the transmission coefficient calculated in model (i) as a function of the reduced energy $E / V_{2}$ for states above the barrier. We have chosen a GaAs-AlGaAs SL as a typical example with the following structural parameters: $d_{1}=200 \AA, d_{2}=15 \AA, V_{2}=0.4 \mathrm{eV}$, and $N=200$. The arrows are located at the energies predicted by relation (26). It turns out that these energies are given by

$$
E=\frac{n^{2} \pi^{2} \hbar^{2}}{2 m_{1} d_{1}^{2}}
$$

$n$ being an integer number. It is clear that they coincide with the sharp resonances in the transmission coefficient that can be observed in the figure.

To check whether the energies given by the previous relation (29) correspond to extended states or not, we represent in Fig. 2 the transmission coefficient for a couple of such energies as a function of the size of the system, and compare it with the case in which the energy of the state lies between two of them. For the energies in Eq. (29) the transmission coefficient remains constant as a function of the size $N$, this behavior being expected for an extended state. Meanwhile, for a state with energy between two resonances the transmission coefficient decays exponentially.

\section{CONCLUSIONS}

In summary, we have shown that two particular models of correlated disordered SL's exhibit delocalized states for electrons as well as for phonons. This result has been demonstrated analytically as well as numerically. We have found exactly the energy and frequency for which extended states 
appear. Notice from Fig. 1 that the resonances of the transmission coefficient around the theoretical values (26) are rather broad. This suggests that electron and phonon states close to the values given by Eq. (26) should display a rather large localization length, even larger than the SL length. This is relevant for transport measurements provided the Fermi level (in the case of electrons) are located close to one of these maxima. In such a case, one would expect an enhance- ment of the dc conductance of the sample, as it was actually observed in the case of the so-called random dimer SL's. ${ }^{22}$

\section{ACKNOWLEDGMENTS}

The work of T.H. and A.S. was supported in part by INTAS, Grant No. 96-524. Work at Madrid was supported by CAM, Grant No. 07N/0034/98.
*Electronic address: sedrak@1x2.yerphi.am; sedrak@alf.nbi.dk

${ }^{1}$ P. W. Anderson, Phys. Rev. 109, 1492 (1958); P. W. Anderson, Phys. Rev. B 23, 4828 (1981).

${ }^{2}$ N. F. Mott and W. D. Twose, Adv. Phys. 10, 107 (1961).

${ }^{3}$ E. Abrahams, P. W. Anderson, D. C. Licciardello, and T. V. Ramakrishnan, Phys. Rev. Lett. 42, 673 (1979).

${ }^{4}$ J. M. Ziman, Models of Disorder (Cambridge University Press, London, 1979).

${ }^{5}$ J. C. Flores, J. Phys.: Condens. Matter 1, 8471 (1989).

${ }^{6}$ D. H. Dunlap, H.-L. Wu, and P. Phillips, Phys. Rev. Lett. 65, 88 (1990).

${ }^{7}$ H.-L. Wu and P. Phillips, J. Chem. Phys. 93, 7369 (1990).

${ }^{8}$ H.-L. Wu and P. Phillips, Phys. Rev. Lett. 66, 1366 (1991); H.-L. Wu and P. Phillips, Science 252, 1805 (1991).

${ }^{9}$ A. Bovier, J. Phys. A 25, 1021 (1992).

${ }^{10}$ S. Gangopadhyay and A. K. Sen, J. Phys.: Condens. Matter 4, 9939 (1992).

${ }^{11}$ J. C. Flores, J. Phys. A 26, L1255 (1993).

${ }^{12}$ P. K. Datta, D. Giri, and K. Kundu, Phys. Rev. B 47, 10727 (1993); 48, 16347 (1993).

${ }^{13}$ F. Domínguez-Adame, E. Maciá, and A. Sánchez, Phys. Rev. B 48, 6054 (1993)

${ }^{14}$ A. Sánchez, E. Maciá, and F. Domínguez-Adame, Phys. Rev. B 49, 147 (1994); A. Sánchez, F. Domínguez-Adame, and E. Maciá, ibid. 51, 173 (1995).

${ }^{15}$ E. Diez, A. Sánchez, and F. Domínguez-Adame, Phys. Rev. B 50, 14359 (1994); F. Domínguez-Adame, A. Sánchez, and E. Diez, ibid. 50, 17736 (1994).
${ }^{16}$ A. Sánchez, F. Domínguez-Adame, G. P. Berman, and F. Izrailev, Phys. Rev. B 51, 6769 (1994).

${ }^{17}$ S. N. Evangelou and D. E. Katsanos, Phys. Lett. A 164, 456 (1992).

${ }^{18}$ S. N. Evangelou and A. Z. Wang, Phys. Rev. B 47, 13126 (1993).

${ }^{19}$ S. N. Evangelou and E. N. Economou, J. Phys. A 26, 2803 (1993).

${ }^{20}$ M. Hilke, J. Phys. A 27, 4773 (1994); M. Hilke and J. C. Flores, Phys. Rev. B 55, 10625 (1997); M. Hilke, J. Phys. A 30, L367 (1997).

${ }^{21}$ J. Heinrichs, Phys. Rev. B 51, 5699 (1995).

${ }^{22}$ V. Bellani, E. Diez, R. Hey, L. Toni, L. Tarricone, G. B. Parravicini, F. Domínguez-Adame and R. Gómez-Alcalá, Phys. Rev. Lett. 82, 2159 (1999).

${ }^{23}$ D. G. Sedrakyan and A. G. Sedrakyan, Phys. Rev. B 60, 10114 (1999).

${ }^{24}$ R. Landauer, Philos. Mag. 21, 863 (1970).

${ }^{25}$ P. W. Anderson, D. J. Thouless, E. Abrahams, and D. S. Fisher, Phys. Rev. B 22, 3519 (1980).

${ }^{26}$ E. Erdós and R. C. Herndon, Adv. Phys. 31, 65 (1982).

${ }^{27}$ A. Abrikosov, Solid State Commun. 37, 997 (1981); V. Melnikov, Fiz. Tverd. Tela (Leningrad) 23, 782 (1981) [Sov. Phys. Solid State 23, 444 (1981)].

${ }^{28}$ G. G. Kozlov, V. A. Malyshev, F. Domínguez-Adame, and A. Rodríguez, Phys. Rev. B 58, 5367 (1998).

${ }^{29}$ J. H. Luscombe, Nanotechnology 4, 1 (1993).

${ }^{30}$ I. Gómez, F. Domínguez-Adame, E. Díez, and V. Bellani, J. Appl. Phys. 85, 3916 (1999). 